\title{
INTERVENÇÃO EM AUTORREGULAÇÃO DA APRENDIZAGEM COM ALUNOS DO ENSINO SUPERIOR: ANÁLISE DA PRODUÇÃO CIENTÍFICA
}

\author{
Danielle Ribeiro Ganda \\ Universidade Estadual de Campinas \\ Evely Boruchovitch \\ Universidade Estadual de Campinas
}

\begin{abstract}
Resumo
Pesquisas recentes na área educacional têm evidenciado altas taxas de reprovação e evasão no Ensino Superior. Estudiosos afirmam que o problema pode estar relacionado às dificuldades de aprendizagem dos alunos no contexto acadêmico. A autorregulação da aprendizagem é uma perspectiva amplamente investigada pelo seu potencial em auxiliar os estudantes a aprender melhor. O presente trabalho objetivou analisar a produção científica de artigos sobre programas de intervenção em aprendizagem autorregulada no Ensino Superior, publicados em âmbitos nacional e internacional, no período de 2004 a 2016. Os resultados revelaram o impacto positivo que os programas exerceram no fortalecimento da capacidade de aprender a aprender dos universitários. Os programas de intervenção, em sua maioria, foram realizados em cursos extraclasses, com grupos grandes de alunos e dados coletados por meio de instrumentos de autorrelato. Observou-se um maior número de pesquisas realizadas internacionalmente, com o predomínio do ensino de estratégias no aprendizado de língua estrangeira.
\end{abstract}

Palavras-chave: autorregulação; ensino superior; produção científica.

\section{SELF-REGULATION OF LEARNING WITH HIGHER EDUCATION STUDENTS: ANALYSIS OF SCIENTIFIC PRODUCTION}

\begin{abstract}
Recent research in education has revealed high rates of failure and dropout in higher education. Scholars claim that the problem may be related to students' learning difficulties in the academic context. Self-regulation of learning is a largely investigated perspective for this potential to help students to learn better. The present study aimed to analyze the scientific production of articles on self-regulated learning intervention programs in Higher Education, published nationally and internationally, from 2004 to 2016. The results revealed the positive impact that the programs had on strengthening the capacity of learn to learn from college students. Most intervention programs were conducted in extraclass courses, with large groups of students and data collected through self-report instruments. There were a greater number of studies conducted internationally, with the predominance of teaching strategies in foreign language learning.
\end{abstract}

Keywords: self-regulation; higher education; scientific production. 


\title{
INTERVENCIÓN IN AUTORREGULACIÓN DEL APRENDIZAJE CON ESTUDIANTES DE ENSINO SUPERIOR: ANÁLISIS DE LA PRODUCCIÓN CIENTÍFICA
}

\begin{abstract}
Resumen
Investigaciones recientes en educación han mostrado altas tasas de fracaso y deserción en la educación superior. Los académicos dicen que el problema puede estar relacionado con las dificultades de aprendizaje de los estudiantes en el contexto académico. La autorregulación del aprendizaje es una perspectiva ampliamente investigada por su potencial para ayudar a los estudiantes a aprender mejor. El presente trabajo tuvo como objetivo analizar la producción científica de artículos sobre programas de intervención de aprendizaje autorregulados en Educación Superior, publicados a nivel nacional e internacional, de 2004 a 2016. Los resultados revelaron el impacto positivo que los programas tuvieron en el fortalecimiento de la capacidad de los estudiantes para aprender y aprender. La mayoría de los programas de intervención se realizaron en cursos extraclase, con grandes grupos de estudiantes y datos recopilados a través de instrumentos de autoinforme. Hubo un mayor número de estudios realizados internacionalmente, con el predominio de estrategias de enseñanza en el aprendizaje de lenguas extranjeras.
\end{abstract}

Palabras clave: autorregulación; educación superior; producción científica.

\section{INTRODUÇÃO}

O ingresso no Ensino Superior é, certamente, um grande obstáculo na vida de vários alunos que, muitas vezes, se inserem em um contexto educacional para o qual não estão preparados. As pesquisas revelam que até mesmo os estudantes que tinham um ótimo desempenho durante os ensinos Fundamental e Médio, frequentemente se surpreendem com os altos níveis de conhecimento, de dedicação e de excelência que se exigem nas universidades (Ferla, Valcke, \& Schuyten, 2009). Refere-se aqui às universidades com boa qualidade de ensino, corpo docente qualificado e com histórico de valorização do tripé - ensino, pesquisa e extensão. No Brasil, teoricamente, seriam esses os pré-requisitos mínimos necessários para o reconhecimento da instituição pelo Ministério da Educação (MEC).

Perante o baixo rendimento e as altas taxas de evasão de alunos universitários, acarretados, em parte, pelas dificuldades desses alunos, importantes pesquisas na área da Educação têm sido realizadas, com o intuito de identificar as particularidades do problema e, sobretudo, desenvolver propostas de promoção da autorregulação que possam auxiliar os estudantes em seu ingresso e na permanência na faculdade (Dembo, 2000; Magalhães, 2012; Rosário, Núñez, \& Pienda, 2006; Zimmerman, Bonner, \& Kovach, 2002). Há trabalhos, inclusive, que buscam formas de ajudar os graduandos tanto como alunos quanto como futuros profissionais mais autorregulados (Ávila, Pranke, \& Frison, 2018; Boruchovitch \& Ganda, 2013; Bzuneck \& Boruchovitch, 2019; Dembo, 2001; Ganda \& Boruchovitch, 2015, 2018, 2019; McKeachie \& Svinicki, 2006; VanderStoep \& Pintrich, 2003).

Em linhas gerais, a autorregulação pode ser definida como o processo de monitorar, de controlar e de refletir sobre o próprio aprendizado (Zimmerman \& 
Schunk, 2011). Alunos autorregulados tendem a ser mais organizados, esforçados, interessados e críticos. Esses estudantes são mais capazes de estabelecer metas de estudo, analisar seu desempenho, persistir diante das dificuldades, assim como identificar os comportamentos que afetam sua aprendizagem, entre outras qualidades (Schunk \& Zimmerman, 2008). No ambiente profissional, os universitários são mais propensos a expandir sua conduta autorregulada, tendo atitudes e pensamentos que favorecem seu trabalho. Caso se tornem professores, o impacto é ainda maior, pois a autorregulação pode ser instruída aos seus futuros alunos, o que poderá auxiliálos em seu processo de aprendizagem, dirimindo possíveis dificuldades que venham a encontrar.

As pesquisas constatam que ser autorregulado é um atributo que favorece a aquisição do conhecimento pelos alunos, não apenas porque eles utilizam boas técnicas de estudo, mas também porque possuem orientações motivacionais, crenças pessoais e emoções que são favoráveis ao aprender. Observa-se, desse modo, que a autorregulação abrange várias dimensões da aprendizagem como, por exemplo, a cognitiva/metacognitiva, a motivacional, a emocional/afetiva, a social, entre outras (Bembenutty \& Karabenick, 2004; VanderStoep \& Pintrich, 2003; Wolters, 2010; Zimmerman \& Schunk, 2008). Tendo em vista fortalecer a aprendizagem autorregulada nas suas diversas áreas, esforços têm sido empreendidos por educadores contemporâneos, no sentido de desenvolver programas de intervenção voltados à autorregulação dos alunos, conforme se expõe a seguir.

Programas de intervenção em autorregulação da aprendizagem: aspectos gerais

De modo geral, os programas instrucionais voltados à promoção da autorregulação convergem em três pontos principais: podem ser implementados no contexto de ensino de um conteúdo, podem ser realizados em quase todos os níveis educacionais e podem ser eficazes para diferentes tipos de alunos (Wolters, 2010). Embora haja diversas iniciativas educacionais voltadas ao ensino de técnicas individuais de estudo ou de estratégias de aprendizagem, um programa só poderá ser considerado promotor da autorregulação, se incorporar em sua proposta as questões metacognitivas e motivacionais dos alunos (Simpson, Hynd, Nist \& Burrell, 1997; Wolters, 2010).

Há uma variedade de projetos de intervenção para a aprendizagem autorregulada que podem ser classificados em relação à sua modalidade de instrução, ao seu formato, ao seu conteúdo e ao seu tempo de duração (Hofer, Yu, \& Pintrich, 1998; Zimmerman \& Tsikalas, 2005). Podem-se somar a essa categorização outros aspectos como, por exemplo, o público-alvo, o número de alunos e a qualificação do instrutor. Uma síntese da classificação dos programas pode ser visualizada na Tabela 1. 
Tabela 1

Classificação de Programas de Intervenção para a Promoção da Autorregulação

\begin{tabular}{|c|c|c|}
\hline Aspecto & Opções & Descrição \\
\hline \multirow[t]{3}{*}{ Modalidade } & Presencial & $\begin{array}{l}\text { Aulas, orientações e } \\
\text { atividades realizadas } \\
\text { presencialmente. Pode incluir } \\
\text { atividades para serem } \\
\text { realizadas em casa. }\end{array}$ \\
\hline & Virtual & $\begin{array}{l}\text { Aulas, orientações e } \\
\text { atividades realizadas em } \\
\text { ambiente virtual. Uso de } \\
\text { plataformas on-line de } \\
\text { ensino e Sites da internet. }\end{array}$ \\
\hline & $\begin{array}{l}\text { Mista (Blended } \\
\text { Learning, Hybrid } \\
\text { Learning) }\end{array}$ & $\begin{array}{c}\text { Integra aulas e atividades } \\
\text { nas duas modalidades: } \\
\text { presencial e virtual. }\end{array}$ \\
\hline \multirow[t]{2}{*}{ Formato } & $\begin{array}{l}\text { Integração curricular } \\
\text { (Programs Integrated) }\end{array}$ & $\begin{array}{c}\text { Realizado dentro do contexto } \\
\text { de uma disciplina específica } \\
\text { do curso universitário. } \\
\text { Atividades incorporadas ao } \\
\text { currículo. }\end{array}$ \\
\hline & $\begin{array}{c}\text { Sobreposição } \\
\text { curricular } \\
\text { (Courses adjunct) }\end{array}$ & $\begin{array}{l}\text { Realizado em horário } \\
\text { extraclasse. Atividades } \\
\text { podem ou não serem } \\
\text { incluídas no currículo. }\end{array}$ \\
\hline \multirow[t]{2}{*}{ Conteúdo } & Específico & $\begin{array}{c}\text { Ensino de estratégias para o } \\
\text { domínio de um conteúdo } \\
\text { específico. }\end{array}$ \\
\hline & Geral & $\begin{array}{c}\text { Ensino de estratégias } \\
\text { passíveis de generalização a } \\
\text { vários tipos de conteúdos e } \\
\text { atividades. }\end{array}$ \\
\hline
\end{tabular}

Tempo de duração

Curta

De 1 a 4 encontros.

Média

De 5 a 12 encontros.

Longa

Acima de 12 encontros.

Público-alvo

Ensino Fundamenta

Crianças, de 7 a 14 anos*.

Ensino Médio

Jovens, de 15 a 17 anos*.

Ensino Superior

Jovens/Adultos, 18 a 30

anos*. 


\begin{tabular}{|c|c|c|}
\hline & $\begin{array}{l}\text { Pós-graduação/ } \\
\text { Profissionais }\end{array}$ & Adultos, acima de 30 anos*. \\
\hline \multirow[t]{4}{*}{ Número de alunos } & Individual & Um aluno por vez. \\
\hline & Grupos pequenos & $\begin{array}{l}\text { De } 2 \text { a } 10 \text { alunos. Podem ser } \\
\text { de cursos e níveis diferentes. }\end{array}$ \\
\hline & Turmas fechadas & $\begin{array}{l}\text { Classes pré-formadas de } \\
\text { alunos. }\end{array}$ \\
\hline & Grupos grandes & $\begin{array}{c}\text { Acima de } 10 \text { alunos. Podem } \\
\text { ser de cursos e níveis } \\
\text { diferentes. }\end{array}$ \\
\hline \multirow[t]{3}{*}{ Qualificação do instrutor } & Professor & $\begin{array}{c}\text { Conduzido por um docente } \\
\text { com domínio na área da } \\
\text { autorregulação. }\end{array}$ \\
\hline & Tutor/Monitor & $\begin{array}{l}\text { Conduzido por um aluno } \\
\text { mais graduado no tema, } \\
\text { geralmente orientado por um } \\
\text { professor. }\end{array}$ \\
\hline & Autoinstruído & $\begin{array}{l}\text { Realizado individualmente, } \\
\text { sem a presença de um } \\
\text { professor ou tutor/monitor. }\end{array}$ \\
\hline
\end{tabular}

Nota. *Faixa etária aproximada, podendo variar conforme contexto de aprendizagem, curso ou região.

Nas últimas décadas, com o avanço das tecnologias e com a expansão da internet, tem havido um acentuado crescimento no uso de recursos tecnológicos e virtuais no processo de ensino (Sun \& Rueda, 2012). Zimmerman e Tsikalas (2005) declaram que os computadores podem constituir uma importante ferramenta no desenvolvimento da aprendizagem autorregulada. Segundo Shen e Liu (2011), o método de ensino que envolva o uso de dispositivos eletrônicos e da internet pode tanto fomentar a autorregulação quanto revelar o nível autorregulatório dos alunos, uma vez que a aprendizagem em ambiente on-line pressupõe maior autonomia e disciplina da pessoa durante o estudo (Sun \& Rueda, 2012).

No que concerne ao conteúdo, os programas de intervenção que visem a uma aquisição duradoura da capacidade de autorregulação devem apresentar diversas estratégias de aprendizagem, passíveis de serem generalizadas para outras matérias e outros contextos de aprendizagem (Hofer et al., 1998; Shen \& Liu, 2011). No decorrer do curso, devem-se propor atividades variadas nas quais os alunos tenham a oportunidade de aplicar as estratégias demonstradas e de testar aquelas que se adaptam ao seu estilo pessoal de aprender (Simpson et al., 
1997). Contudo, cabe ressaltar que, embora o conhecimento das estratégias seja de suma importância para a autorregulação, ele por si só não garante a sucesso da aprendizagem.

Os alunos necessitam de saber quais são as estratégias, como e em quais atividades usá-las e, até mesmo, quando modificá-las, caso percebam que não estão sendo eficazes para o alcance dos seus objetivos (Boruchovitch, Costa, \& Neves, 2005; Pozo, 1996). Além disso, para que elas de fato sejam aplicadas durante a realização de uma atividade, é necessário que outros aspectos pessoais do estudante sejam considerados como, por exemplo, a sua metacognição, a sua autoeficácia, a sua motivação, as suas crenças atribucionais e, inclusive, a sua percepção sobre o ambiente de aprendizagem (Brunstein \& Glaser, 2011; Weiner, 2010; Zimmerman et al., 2002).

Embora os educadores tenham sistematicamente reafirmado a importância de se fomentar a autorregulação no ambiente educacional, ainda se observa uma lacuna no ensino de estratégias autorregulatórias no Ensino Superior (Wolters, 2010; Zimmerman \& Schunk, 2008). Por meio de um trabalho que estimule cada uma das áreas do conhecimento supracitadas, acredita-se que os alunos possam fortalecer sua aprendizagem, tornando-se universitários e futuros profissionais mais autônomos, motivados e autorreflexivos, ou seja, mais autorregulados.

Desse modo, tendo em vista mapear o estado da arte sobre o tema, efetuou-se uma busca na literatura científica internacional e nacional a respeito de trabalhos científicos experimentais e quase-experimentais que tenham investigado a aprendizagem autorregulada, de modo geral, e as variáveis a ela relacionadas. Especificadamente, objetivou-se analisar as características principais dos programas de intervenção voltados à promoção, à autorregulação e as possíveis contribuições das pesquisas.

\section{MÉTODO}

A pesquisa foi realizada nas bases de dados on-line e periódicos eletrônicos da American Psychological Association (APA), Scientific Library On Line (SciELO), Education Resources Information Center (ERIC), Science Direct e Biblioteca Virtual em Saúde (BVS-Psi), além de periódicos impressos disponíveis nas bibliotecas da Universidade Estadual de Campinas - UNICAMP. O período de abrangência foi de 2004 a 2016. Para a investigação internacional, foram usados os seguintes descritores: "self-regulation", "self-regulated learning", "intervention", "self-regulated strategies", "college students" e "pre-service teachers". Para a pesquisa nacional, foram usados os termos: "autorregulação", "aprendizagem autorregulada", "intervenção", "estratégias de autorregulação", "alunos universitários", "estudantes de pedagogia" e "formação de professores". Deve-se ressaltar que aqui foram incluídos apenas os estudos publicados em 
artigos científicos e não os trabalhos apresentados em dissertações, teses e livros.

Durante a busca, encontraram-se diversos estudos sobre autorregulação de caráter teórico e descritivo-correlacional, além de pesquisas conduzidas em diferentes áreas do conhecimento, como, por exemplo, a organizacional, a esportiva e a clínica. Contudo, considerando os objetivos do presente estudo, os critérios de inclusão na revisão de literatura abarcaram apenas os estudos realizados no contexto do Ensino Superior e de caráter experimental ou quaseexperimental. Embora tenham sido encontrados trabalhos de intervenção realizados com alunos da Educação Infantil, dos Ensinos Médio, Fundamental e, inclusive, com profissionais já graduados, são aqui relatadas somente as pesquisas com estudantes universitários. Na Tabela 2 encontra-se um panorama geral de todos os trabalhos encontrados na busca científica.

Tabela 2

Levantamento de Estudos para a Promoção da Aprendizagem Autorregulada: Pesquisas Experimentais e Quase-experimentais no contexto educacional

\begin{tabular}{|c|c|c|}
\hline Amostra & Autoria / Ano de publicação & Local de realização \\
\hline $\begin{array}{c}\text { Alunos da } \\
\text { Educação Infantil }\end{array}$ & $\begin{array}{c}\text { Ávila \& Frisson, } 2012 \\
\text { Roque, } 2012 \\
\text { Rosário, Barbosa, Núnez, \& } \\
\text { González-Pienda, } 2005\end{array}$ & $\begin{array}{c}\text { Brasil (RS) } \\
\text { Portugal } \\
\text { Portugal }\end{array}$ \\
\hline $\begin{array}{l}\text { Alunos do Ensino } \\
\text { Fundamental }\end{array}$ & $\begin{array}{c}\text { Bilimória \& Almeida, } 2008 \\
\text { Brunstein \& Glaser, } 2011 \\
\text { Burke \& Williams, 2008, } 2012 \\
\text { Costa \& Boruchovitch, } 2009 \\
\text { Cleary, Platten, \& Nelson, } 2008 \\
\text { Gago, } 2012 \\
\text { Gomes \& Boruchovitch, } 2011 \\
\text { Lizarraga, Baquedano, Magado, \& } \\
\text { Elawar, 2012 } \\
\text { Perels, Gurtler, \& Schmitz, } 2005 \\
\text { Peters \& Kitsantas, } 2009 \\
\text { Punhagui \& Souza, 2012 } \\
\text { Redford, Thiede, Wiley \& Griffin, } \\
\text { 2012 } \\
\text { Rosário, González-Pienda, Núnez \& } \\
\text { Mourão, 2005 }\end{array}$ & $\begin{array}{c}\text { Portugal } \\
\text { Alemanha } \\
\text { Escócia } \\
\text { Brasil (GO) } \\
\text { Estados Unidos } \\
\text { Portugal } \\
\text { Brasil (SP) } \\
\text { Espanha } \\
\text { Alemanha } \\
\text { Estados Unidos } \\
\text { Brasil (PR) } \\
\text { Estados Unidos } \\
\text { Portugal }\end{array}$ \\
\hline $\begin{array}{c}\text { Alunos do Ensino } \\
\text { Médio }\end{array}$ & $\begin{array}{c}\text { Abreu, } 2012 \\
\text { Chung \& Yuen, } 2012 \\
\text { Cleary \& Zimmerman, } 2004 \\
\text { Kostons, Gog, \& Paas, } 2012\end{array}$ & $\begin{array}{c}\text { Portugal } \\
\text { Hong Kong } \\
\text { Estados Unidos } \\
\text { Holanda }\end{array}$ \\
\hline
\end{tabular}




\begin{tabular}{|c|c|c|}
\hline $\begin{array}{l}\text { Alunos do Ensino } \\
\text { Superior }\end{array}$ & $\begin{array}{c}\text { Alexiou \& Paraskeva, } 2010 \\
\text { Bembenutty \& White, } 2013 \\
\text { Bernacki, Byrnes \& Cromley, } 2012 \\
\text { Bown, } 2009 \\
\text { Boruchovitch \& Ganda, } 2013 \\
\text { Cotterall \& Murray, } 2009 \\
\text { Dunlosk \& Rawson, } 2012 \\
\text { Fabriz, Ewijk, Poarch \& Buttner, } 2013 \\
\text { Fantinel, Angelo, Lima \& Maraschin, } \\
\text { 2013 } \\
\text { Frison \& Moraes, 2010 } \\
\text { Frison \& Veiga Simão, } 2011 \\
\text { Goh, Seet \& Chen, 2012 } \\
\text { Granville \& Dison, 2005 } \\
\text { Johnson, Archibald \& Tenenbaum, } \\
2010 \\
\text { Kauffman, Zhao \& Yang, } 2011 \\
\text { Leutner, Leopold, \& Elzen-Rump, } \\
\text { 2007 } \\
\text { Matsumoto, Hiromori, \& Nakayama, } \\
\text { 2013 } \\
\text { Nuckels, Hubner, \& Renkl, } 2009 \\
\text { Rosário et. al., 2010 }\end{array}$ & $\begin{array}{c}\text { Grécia } \\
\text { Estados Unidos } \\
\text { Estados Unidos } \\
\text { Estados Unidos } \\
\text { Brasil (SP) } \\
\text { Japão } \\
\text { Estados Unidos } \\
\text { Alemanha } \\
\text { Brasil (RS) } \\
\text { Brasil (RS) } \\
\text { Brasil (RS) e Portugal } \\
\text { Nova Zelândia } \\
\text { África do Sul } \\
\text { Estados Unidos } \\
\text { Estados Unidos } \\
\text { Alemanha } \\
\text { Japão } \\
\text { Alemanha } \\
\text { Portugal e Espanha }\end{array}$ \\
\hline $\begin{array}{l}\text { Profissionais } \\
\text { graduados }\end{array}$ & $\begin{array}{l}\text { Bailey, Dunlosky, \& Hertzog, } 2010 \\
\text { Glava \& Glava, } 2010 \\
\text { Randi, Corno, \& Johnson, } 2011 \\
\text { Sitzmann, Bell, Kraiger, \& Kanar, } \\
2009 \\
\text { Sitzmann \& Ely, } 2010 \\
\text { Sitzmann \& Johnson, } 2012\end{array}$ & $\begin{array}{l}\text { Estados Unidos } \\
\quad \text { Romênia } \\
\text { Estados Unidos } \\
\text { Estados Unidos } \\
\text { Estados Unidos } \\
\text { Estados Unidos }\end{array}$ \\
\hline
\end{tabular}

A apresentação dos trabalhos segue a ordem cronológica, do mais antigo para o mais atual; relata-se, primeiramente, a produção científica internacional e, em seguida, a nacional. A descrição das publicações foi realizada de modo sucinto, ressaltando as informações mais importantes de cada trabalho como: o objetivo geral, a proposta de intervenção, o método de coleta de dados e os principais resultados.

\section{RESULTADOS}

Estudos da literatura internacional

Os professores Granville e Dison (2005) desenvolveram um projeto ao longo de um ano letivo, com o objetivo de desenvolver as habilidades metacognitivas dos alunos, tendo em vista o aprimoramento dos processos de 
aprendizagem e dos pensamentos de ordem superior, com uma amostra de 35 estudantes ingressantes de uma universidade africana. A partir da análise preliminar de entrevistas semiestruturadas realizadas durante o curso, os autores observaram que o pensamento metacognitivo constituiu uma importante ferramenta para a aprendizagem. Houve também evidências de melhora da consciência metacognitiva e dos pensamentos analíticos e autoavaliativos dos universitários (Granville \& Dison, 2005).

A partir de um estudo experimental, Leutner, Leopold e Elzem-Rump (2007) investigaram a eficácia de um programa de treinamento computadorizado de quatro semanas voltado ao uso de estratégias cognitivas para a promoção da autorregulação de 45 alunos universitários da Alemanha. A coleta de dados foi realizada por meio de uma escala de inteligência verbal, um teste de conhecimento sobre o conteúdo do programa, um teste de compreensão textual e uma breve redação sobre o uso de estratégias. Os resultados revelaram que os alunos do grupo experimental se mostraram mais capazes de aplicar a estratégia cognitiva em consonância com seus objetivos, tanto durante a leitura como na recuperação das informações do texto estudado (Leutner et al., 2007).

Bown (2009) buscou identificar os comportamentos autorregulatórios dos alunos durante o aprendizado de língua estrangeira (russo), realizado durante um ano letivo, por meio de um Programa de Autoinstrução. Participaram do estudo vinte alunos de uma universidade americana que responderam entrevistas semiestruturadas e escreveram diários narrativos ao longo do curso. Os alunos relataram que estudar pelo método autoinstrucional foi um desafio, devido à ausência de uma estrutura e de cobrança externa feita por um professor. Tendo em vista suprir essas carências, os alunos aplicaram diversas estratégias cognitivas para regular sua própria aprendizagem como, por exemplo, planejar, traçar metas de curto prazo, procurar materiais extras, entre outros (Bown, 2009).

Os pesquisadores Nuckels, Hubner e Renkl (2009) desenvolveram um estudo para avaliar o efeito que os avisos (prompts), uma estratégia autoguiada de escrita, teriam para a compreensão dos alunos durante a redação de protocolos. No estudo realizado por três meses, participaram 103 estudantes de uma universidade alemã que responderam questões abertas de compreensão do conteúdo no pré-teste e no pós-teste. Os resultados revelaram que aplicar a estratégia de dar avisos apoiando todas as fases da autorregulação foi a melhor condição para se fomentar a compreensão (Nuckels et al., 2009).

Em uma pesquisa realizada com 269 alunos de uma universidade japonesa, ao longo de 15 semanas, Cotterall e Murray (2009) analisaram o progresso dos estudantes em um curso autodirigido para aprendizado da Língua Inglesa. Nas etapas de pré-teste e de pós-teste, os alunos responderam a um questionário sobre o domínio na língua com dez itens em escala do tipo Likert de cinco pontos. Os resultados revelaram que o curso autodirigido contribuiu para o 
aumento das crenças positivas dos alunos, especialmente no que concerne ao conhecimento metacognitivo e ao controle aplicado na aprendizagem de línguas.

Os professores Johnson, Archibald e Tenenbaum (2010) realizaram dois estudos, cada um com dez semanas de duração, para mensurar os efeitos de um método de aprendizagem por anotações on-line no desenvolvimento das habilidades de compreensão da leitura, pensamento crítico e metacognição de alunos de uma universidade americana. O método denominado de Social Annotation Model - Learning System (SAM-LS) combinou o uso de estratégias instrucionais, aprendizado em grupo e a ferramenta de aprendizagem colaborativa de anotação social em ambiente virtual: HyLigther. Os estudos $01 \mathrm{e}$ 02 envolvendo, respectivamente, 254 e 267 universitários americanos, mostraram que as habilidades referentes à compreensão da leitura e à metacognição dos alunos aumentaram quando eles trabalharam em grupo (Johnson et al., 2010).

Em uma parceria entre pesquisadores da Espanha e Portugal, Rosário et al. (2010) implementaram uma proposta de intervenção voltada para o desenvolvimento de estratégias de autorregulação da aprendizagem, com 176 alunos ingressantes de cursos de Educação de duas universidades, de ambos os países. A proposta baseou-se no programa de autorregulação intitulado "Cartas do Gervásio a seu Umbigo", de Rosário, Núñez e Pienda, 2006. Os dados foram coletados por meio de questionários, inventários uma escala Likert. De maneira geral, os resultados indicaram que o programa de intervenção foi eficaz para a promoção da autorregulação da aprendizagem. Os alunos aprimoraram o conhecimento sobre as estratégias de aprendizagem, melhoraram as suas competências autorregulatórias e reduziram o uso de abordagens superficiais durante o aprendizado (Rosário et al., 2010).

O uso de portfólios on-line como uma possível ferramenta eficaz para a promoção da aprendizagem autorregulada foi a questão suscitada por Alexiou e Paraskeva (2010). O seu estudo, realizado com 41 estudantes do Curso de Ciências da Computação de uma universidade da Grécia. Os alunos responderam um questionário a respeito do uso de estratégias de aprendizagem e três escalas sobre autoavaliação do portfólio pessoal, engajamento na atividade e automonitoramento. Durante um semestre, os participantes realizaram semanalmente atividades individuais programadas com o uso da ferramenta virtual de portfólios. Os universitários relataram que o uso de portfólio on-line favoreceu a compreensão e o engajamento durante as atividades e que se configurou como uma importante ferramenta para aprimorar as suas habilidades de aprendizagem autorregulatórias (Alexiou \& Paraskeva, 2010).

Kauffman, Zhao e Yang (2011) realizaram dois estudos para examinar como duas estratégias designadas para aprimorar a autorregulação (a. diferentes métodos de anotação e b. lembretes de automonitoramento), poderiam influenciar a quantidade de informação adquirida e o desempenho de alunos 
universitários americanos, em um contexto de aprendizagem on-line. A amostra total dos estudos incluiu 149 alunos matriculados em disciplinas de Psicologia Educacional, que responderam questionários de autorrelato e testes de conhecimento. Os resultados revelaram que os alunos que utilizaram os métodos de anotação mais elaborados obtiveram melhor sistematização do conteúdo e que resultaram em um conjunto de anotações mais completo levando a um desempenho superior (Kauffman et al., 2011).

A relação entre as metas de aprendizagem, o uso de estratégias cognitivas e o desempenho foi investigada por Bernacki, Byrnes e Cromley (2012) com 160 universitários americanos de cursos de Educação que responderam um questionário de metas de aprendizagem, um instrumento sobre o conhecimento prévio mais a compreensão leitora antes e uma tarefa de aprendizagem. De modo geral, os dados indicaram que os tipos de metas de aprendizagem influenciaram os comportamentos de aprendizagem autorregulados e alguns desses comportamentos, como o sublinhar e monitorar, tiveram uma correlação positiva com a compreensão da leitura. Os estudantes com metas de aprendizagem do tipo domínio/aprender usaram mais as estratégias de busca de informações, de anotação e de monitoramento. Já os alunos com meta do tipo performance-evitação apresentaram uma menor tendência no uso das duas primeiras estratégias mencionadas (Bernacki et al., 2012).

Os pesquisadores Goh, Seet e Chen (2012) realizaram um estudo experimental para verificar o impacto que um sistema de intervenção por mensagens de celular (SMS) persuasivas causaria no uso de estratégias de aprendizagem autorregulatórias. A pesquisa foi realizada com 57 alunos de uma universidade da Nova Zelândia, matriculados em uma disciplina introdutória sobre sistema da informação, com duração de 12 semanas. Os universitários responderam à uma escala Likert sobre motivação e estratégias de aprendizagem. O estudo revelou um impacto positivo do método de intervenção por mensagens de celular na aprendizagem no que tange, principalmente, os seguintes aspectos: gerenciamento de tempo, estabelecimento de metas, percepção de valor da tarefa e uso de estratégias cognitivas e metacognitivas. O envio de SMS mostrou-se uma técnica viável e eficiente para estimular, manter e melhorar o uso de estratégias de aprendizagem e o desempenho dos participantes. Observou-se que os alunos com pior rendimento acadêmico foram os que mais se beneficiaram da intervenção, obtendo uma melhora ainda mais significativa do que os colegas (Goh et al., 2012).

Dunlosk e Rawson (2012) conduziram dois estudos, com um total de 198 universitários americanos, para analisar a relação entre a precisão no monitoramento metacognitivo e a recuperação da informação (memória) no processo de aprendizagem autorregulada. A coleta de dados foi realizada com testes de conhecimento e atividades metacognitivas. Os resultados revelaram que a precisão no julgamento metacognitivo favoreceu a retenção da informação 
e a aprendizagem efetiva, quando foi dada ao aluno a possibilidade de rever seus erros e acertos em uma tarefa e de estudar novamente os conceitos que não tinha dominado. Observou-se, também, que os universitários menos confiantes quanto ao acerto das questões dedicaram mais tempo ao reestudo do conteúdo, o que desencadeou uma memorização mais e um aprendizado mais efetivo (Dunlosk \& Rawson, 2012).

Os professores Matsumoto, Hiromori e Nakayama (2013) desenvolveram um curso de instrução em estratégias autorreguladas de leitura, com o objetivo de influenciar a motivação e as crenças gerais de aprendizagem de 360 alunos ingressantes de uma universidade japonesa, no aprendizado de uma segunda língua (Inglês). Os universitários preencheram uma escala composta por três subescalas: uso de estratégias de leitura, motivação e crenças de aprendizagem. Os resultados mostraram que os tipos de motivação afetam consideravelmente o modo pelo qual o uso de estratégias e as crenças gerais de aprendizagem se relacionam. O curso de instrução em estratégias de leitura teve efeitos positivos e facilitadores não apenas no uso das estratégias para a compreensão textual, como também na motivação e nas crenças dos alunos no contexto de aprendizado de uma segunda língua. A estratégia "seleção da ideia principal" foi a que revelou maior influência significativa e positiva na compreensão, na motivação e nas crenças dos estudantes (Matsumoto et al., 2013).

A pesquisa de Fabriz, Ewijk, Poarch e Buttner (2013) teve como objetivo investigar os efeitos do uso do diário de aprendizagem no desenvolvimento da autorregulação e no desempenho acadêmico, ao longo de um semestre, por 77 alunos de uma universidade alemã, matriculados numa disciplina de Autorregulação e outra de Gramática. Os participantes responderam uma escala sobre o uso de estratégias de aprendizagem (cognitivas, metacognitivas e de administração de recursos) e um teste de conhecimento. Os alunos que participaram da disciplina teórica sobre a autorregulação e, ao mesmo tempo, fizeram o diário de aprendizagem obtiveram aumento no controle da atenção e no senso de autoeficácia e diminuição no estresse e nas emoções negativas. Entretanto, os universitários da disciplina de gramática que fizeram o diário relataram diminuição na motivação intrínseca e nas emoções positivas relacionadas à aprendizagem. De acordo com os pesquisadores, esse dado revela a importância de não apenas usar uma boa estratégia de estudo no contexto acadêmico, mas, sobretudo, de explicar ao aluno os benefícios do automonitoramento e da autorregulação para a sua aprendizagem (Fabriz et al., 2013).

Assim como o diário de aprendizagem, outra ferramenta de automonitoramento para a promoção da aprendizagem autorregulada é o Homework log, que é um quadro no qual o aluno registra as metas, as distrações, o tempo despendido e até o nível de satisfação ao realizar as atividades para casa. Com o uso dessa ferramenta, Bembenutty e White (2013) 
avaliaram a relação entre as crenças motivacionais, as estratégias de pedir ajuda, as práticas individuais de realização de tarefa de casa, a satisfação e o desempenho acadêmico de alunos em universidade nos Estados Unidos. A amostra foi composta por 133 universitários que responderam a três escalas tipo Likert referentes às variáveis pedir ajuda, autoeficácia para a aprendizagem e motivação intrínseca. Os resultados indicaram uma correlação positiva entre a satisfação, o desempenho acadêmico, os esforços na realização da tarefa, a tendência adaptativa da estratégia de pedir ajuda e as notas finais obtidas no curso.

Em resumo, na literatura internacional, foram encontrados, ao todo, quinze trabalhos, a maioria realizada nos Estados Unidos. A busca revelou um expressivo número de pesquisas que tiveram como foco a promoção da autorregulação e o ensino de estratégias destinadas ao aprendizado de língua estrangeira. Os programas, em sua maioria, foram realizados em cursos de sobreposição curricular, ou seja, fora das disciplinas regulares do curso universitário, e para grupos grandes de alunos (mais de 15 participantes). Além disso, os modelos de intervenção mais empregados incorporaram o uso de computadores e de atividades on-line e foram realizados em um tempo de média a longa duração (de quatro semanas a um semestre). Nas coletas de dados, houve um predomínio da utilização de instrumentos de autorrelato, embora diversos estudos tenham incorporado a aplicação de testes avaliativos de conteúdo e atividades de mensuração do uso de estratégias de aprendizagem nos momentos em que ocorre.

As pesquisas estrangeiras evidenciaram que diferentes projetos de intervenção podem ser eficazes para a promoção da autorregulação da aprendizagem. Os trabalhos que obtiveram melhores resultados foram aqueles nos quais se investiu em desenvolver conjuntamente diversos componentes cognitivos, metacognitivos e motivacionais da autorregulação. Observa-se, contudo, que poucas pesquisas analisaram os aspectos emocionais que interferem na aprendizagem como, por exemplo, a ansiedade, o estresse, entre outros.

\section{Estudos da literatura nacional}

Frison e Moraes (2010) investigaram em que medida a prática da monitoria auxiliaria os alunos de uma universidade pública do Rio Grande do Sul nos seus processos de autorregulação da aprendizagem. Fizeram parte do estudo 22 acadêmicos, sendo oito monitores, doze alunos que usufruem da monitoria e dois professores-supervisores. De modo geral, os dados revelaram que a prática de monitoria ajudou os alunos a estabelecerem metas, a desenvolverem estratégias e a criarem condições para a realização da aprendizagem.

Em uma parceria entre Brasil e Portugal, Frison e Veiga Simão (2011) desenvolveram um trabalho para analisar a relação entre as narrativas 
autobiográficas e a aprendizagem autorregulada de futuros professores. A amostra foi composta por quinze alunos portugueses, de um curso de formação inicial em Educação Básica, e doze universitários brasileiros, de um curso de Pedagogia do Rio Grande do Sul. Os participantes registraram as suas experiências de aprendizado em portfólios reflexivos, que indicaram importantes contribuições do trabalho, considerando o aumento das aprendizagens, 0 desenvolvimento de competências e as mudanças no nível conceitual e atitudinal pelos alunos.

Fantinel, Angelo, Lima e Maraschin (2013) verificaram o impacto de uma proposta de intervenção voltada à promoção da aprendizagem autorregulada de 41 alunos do primeiro e do quarto semestres do curso de Licenciatura em Matemática a Distância de uma universidade pública do Rio Grande do Sul. Os resultados preliminares revelaram que os alunos se apropriaram de algumas das estratégias autorregulatórias trabalhadas e as mais citadas foram: anotações, elaboração de resumos, uso de cronogramas para gestão do tempo, estudo em grupo, redução da procrastinação, delineamento de objetivos, entre outros.

Boruchovitch e Ganda (2013) realizaram o estudo com 26 alunos do segundo ano do curso de Pedagogia de uma universidade pública de São Paulo. A pesquisa foi realizada ao longo de um semestre letivo, nas quais foram trabalhados importantes tópicos relacionados à autorregulação tais como: autoeficácia, estratégias de aprendizagem, motivação para aprender, entre outros. No início e no fim da disciplina, os alunos responderam a instrumentos de autorrelato e escalas. Os resultados revelaram que, de maneira geral, a proposta de ensino foi eficaz para a promoção das habilidades autorregulatórias.

Em síntese, pode-se verificar que o número de pesquisas voltadas à promoção da aprendizagem autorregulada realizadas no Brasil ainda é incipiente, com apenas quatro publicações, quando se compara aos da literatura internacional. Contudo, os resultados por elas encontrados são animadores, pois revelam os inegáveis benefícios de se investir em propostas que visem ajudar os alunos universitários. A maior parte das propostas de intervenção foi realizada em cursos presenciais extraclasse, com grupos grandes de alunos (acima de quinze participantes) e com foco no ensino de estratégias de aprendizagem. Nos trabalhos, a maioria das intervenções foi conduzida por monitores/tutores sob a orientação de professores e houve o predomínio de instrumentos de autorrelato. Observou-se, ainda, que práticas relativamente simples como, por exemplo, a monitoria e o uso de portfólios foram capazes de contribuir para fomentar a autorregulação dos alunos universitários.

\section{CONSIDERAÇÕES FINAIS}

O presente trabalho visou a fazer um levantamento das pesquisas sobre programas de intervenção em autorregulação da aprendizagem com alunos do 
Ensino Superior, realizadas na última década. De modo geral, observou-se maior quantidade de artigos publicados internacionalmente, com o predomínio do ensino de estratégias voltadas à promoção da autorregulação no aprendizado de língua estrangeira. Contudo, constatou-se que tem ocorrido uma mudança no cenário nacional, visto que, desde 2010, os pesquisadores brasileiros começaram a divulgar seus trabalhos, nos quais têm obtido resultados muito favoráveis ao fortalecimento dos processos autorregulatórios de estudantes universitários.

Além disso, embora não tenha sido o foco desse estudo, notou-se que há, inclusive, a publicação de pesquisas realizadas com alunos desde os primeiros anos do Ensino Fundamental. Essas iniciativas evidenciam que as habilidades cognitivas, metacognitivas e autorregulatórias podem ser fomentadas desde o início da escolarização, o que, possivelmente, promoveria uma aprendizagem de maior qualidade e preveniria diversos problemas educacionais enfrentados pelos estudantes nos anos escolares posteriores (Bandura, 2003; Brunstein \& Glaser, 2011; Boruchovitch et al., 2005; Costa \& Boruchovitch, 2009; Ganda \& Boruchovitch, 2015, 2018, 2019; Gomes \& Boruchovitch, 2011; Rosário et al.; 2007, 2008; Zimmerman \& Schunk, 2008).

No entanto, percebe-se que, muitas vezes, os próprios professores desses alunos não têm conhecimento teórico e prático sobre a autorregulação e variáveis a ela relacionadas como, por exemplo, a motivação, as atribuições causais, as estratégias de aprendizagem, entre outras, que exercem um papel crucial no processo de ensino e aprendizado (Berry, 2006; Martini \& DelPrette, 2002, 2009; Warfield, Wood, \& Lehman, 2005; Woolfson \& Brady, 2009; Salonen, Vauras, \& Efklides, 2005). Os pesquisadores afirmam que há professores que, durante sua formação docente, não tiveram contato com essa fundamentação teórico-metodológica e que, inclusive, não foram estimulados a refletir sobre sua conduta e a aplicar as mesmas estratégias ao longo de sua trajetória acadêmica e profissional (Brunstein \& Glaser, 2011; Dembo, 2001; Marini \& Boruchovitch, 2014; McKeachie \& Svinicki, 2006).

Destaca-se, desse modo, a importância de se fomentar a autorregulação no Ensino Superior, especialmente em cursos de formação docente, como a Pedagogia e as Licenciaturas (Cleary, 2011; Boruchovitch \& Ganda, 2013; Lee, Lim, \& Grabowski, 2010; McKeachie, Lin, \& Middleton, 2004; Middleton, Abrams, \& Seaman, 2011; Schunk \& Zimmerman, 2008). A formação holística dos alunos universitários é, certamente, um modo de favorecer o ensino da próxima geração de estudantes, abrindo os caminhos em prol de uma aprendizagem mais ativa, autônoma e significativa, ou seja, autorregulada. 


\section{REFERÊNCIAS}

Abreu, F.A.R. (2012). Autorregulação da aprendizagem em biologia no ensino secundário: Um estudo sobre os processos de aprendizagem na unidade Obtenção de matéria - heterotrofia e autotrofia do $10^{\circ}$ ano de escolaridade. Relatório de estágio. Instituto de Educação, Universidade do Minho. Braga, Portugal.

Alexiou, A. \& Paraskeva, F. (2010). Enhancing self-regulated learning skills through the implementation of an e-portfolio tool. Innovation and Creativity in Education, 2(2), 3048- 3054.

Ávila, L.T.G., \& Frisson, L.M.B. (2012). Educação física na educação infantil: Estratégias para promover a aprendizagem autorregulada. Revista de Educação PUC-Campinas, 17(2), 181-189.

Ávila, L.T.G.; Pranke, A.; \& Frison, L.M.B. (2018). O uso de estratégias autorregulatórias para aprender e para ensinar: Formação de professores. Perspectiva, 36(4), 1265-1280.

Bailey H, Dunlosky J, Hertzog C. (2010). Self-regulation training at home: Does it improve older adults' learning? Gerontology, 56, 414-420.

Bandura, A. (2003). Observational learning. In J. H. Byrne, Encyclopedia of Learning and Memory, Nova York, NY: Macmillan, p. 482-484.

Bembenutty, H., \& Karabenick, S. A. (2004). Inherent association between academic delay of gratification, future time perspective, and self-Regulated learning. Educational Psychology Review, 16(1), 35-57.

Bembenutty, H., \& White, M. C. (2013). Academic performance and satisfaction with homework completion among college students. Learning Individual Differences, 24, 83-88. doi:10.1016/j.lindif.2012.10.013

Berry, R. A. W. (2006). Beyond strategies: Teacher beliefs and writing instruction in two primary inclusion classroom. Journal of Learning Disabilities, 39(1), 11-24.

Bernacki, M., Byrnes, J., \& Cromley, J. (2012). The effects of achievement goals and self-regulated learning behaviors on reading comprehension in technologyenhanced learning enviroments. Contemporary Educacional Psychology 37, 148-161. doi:10.1016/j.cedpsych.2011.12.001

Bilimória, H. \& Almeida, L. (2008). Aprendizagem auto-regulada: Fundamentos e organização do programa SABER. Revista Semestral da Associação Brasileira de Psicologia Escolar e Educacional (ABRAPEE), 12(1), 13-22.

Boruchovitch, E., Costa, E. R., \& Neves, E. R. C. (2005). Estratégias de aprendizagem: Contribuições para a formação de professores nos cursos superiores. In M. C. R. A. Joly, A. A. A. Santos, \& F. F. Sisto (Eds.), Questões do cotidiano universitário, (p.239-260). São Paulo, SP: Casa do Psicólogo. 
Boruchovitch, E., \& Ganda, D. R. (2013). Fostering self-regulated skills in an educational psychology course for Brazilian preservice teachers. Journal of Cognitive Educacional Psychology 12, 157-177. doi:10.1891/19458959.12.2.157

Bown, J. (2009). Self-regulatory strategies and agency in self-instructed language learning: A situated view. Modern Language Journal, 93(4), 570583.

Brunstein, J. C., \& Glaser, C. (2011). Testing a path-analytic mediation model of how self-regulated writing strategies improve fourth graders' composition skills: A randomized controlled trial. Journal of Educacional Psychology, 103, 922-938. doi: $10.1037 /$ a0024622

Burke, L. A.; \& Williams, J. M. (2008). Developing young thinkers: An intervention aimed to enhance children's thinking skills. Thinking Skills and Creativity, 3, 104-124.

Burke, L. A.; \& Williams, J. M. (2012). The impact of a thinking skills intervention on children's concepts of intelligence. Thinking Skills and Creativity, 7, 145152.

Bzuneck, J. A.; \& Boruchovitch, E. (2019). Motivação de estudantes no Ensino Superior: Como fortalecê-la?. In L. Almeida (Ed.), Motivação de estudantes no ensino superior: Como fortalecê-la?. Braga: Adipsieduc, p. 137-157.

Cleary, T. J. (2011). Professional development needs and practices among educators and school psychologists. New Direction Teaching Learning 126, 77-87. doi:10.1002/ tl.446

Chung, Y.B. \& Yuen, M. (2012). Students' perceptions of a reporting and feedback system for learning and development in an 'inviting school' in Hong Kong. Pastoral Care in Education, 30(3), 241-262.

Cleary, T.J., \& Zimmerman, B.J. (2004). Self-regulation empowerment program: A school-based program to enhance self-regulated and self-motivated cycles of student learning. Psychology in the Schools, 41(5), 537-550.

Costa, E. R., \& Boruchovitch, E. (2009). As estratégias de aprendizagem e a produção de textos narrativos. Psicologia. Reflexão e Crítica 22, 173-180. doi: 10.1590/S0102-79722009000200002

Cotterall, S., \& Murray, G. (2009). Enhancing metacognitive knowledge: Structure, affordances and self. System, 37, 34-45.

Cleary, T.J., Platten, P., \& Nelson, A. (2008). Effectiveness of the self-regulation empowerment program with urban high school students. Journal of advanced academics, 20(1), 70-107.

Dembo, M. (2000). Motivation and learning strategies for college success: A selfmanagement approach. Mahwah, NJ: Erlbaum Publishers.

Dembo, M. H. (2001). Learning to teach is not enough: future teachers also need to learn to learn. Teacher Educacion Quarterly 28(4), 23-35. 
Dunlosk, J., \& Rawson, K. A. (2012). Overconfidence produces underachievement: Inaccurate self evaluations undermine students' learning and retention. Learning and Instruction, 22, 271-280.

Fabriz, S., Ewijk, A. D., Poarch, G., \& Buttner, G. (2013). Fostering selfmonitoring of university students by means of a standardized learning journal-a longitudinal study with process analyses. European Journal Psychology of Education 29, 239-255. doi:10.1007/s10212-013-0196-z

Fantinel, P. C., Angelo, N. P., Lima, J. V., \& Maraschin, C. (2013). Autorregulação da aprendizagem: Uma competência fundamental na formação do licenciado em matemática à distância. Belém, PA: Anais do X Congresso Brasileiro de Ensino Superior à Distância.

Ferla, J., Valcke, M., \& Schuyten, G. (2009). Student models of learning and their impact in study strategies. Studies in Higher Education, 34(2), 185202.

Frison, L.M.B.; \& Moraes, M.A.C. (2010). As práticas de monitoria como possibilitadoras dos processos de autorregulação das aprendizagens discentes. Poiésis Pedagógica, 8(2), 144- 158.

Frison, L.M.B., \& Veiga Simão, A.M. (2011). Abordagem autobiográfica Narrativas de formação e de autorregulação da aprendizagem reveladas em portfólios reflexivos. Educação, 34(2), 198-206.

Gago, S.M.F. (2012). Vale a pena reflectir quando se aprende online? Autoregulação em e-English. (Dissertação de Mestrado). Universidade Nova de Lisboa, Faculdade de Ciências Sociais e Humanas.

Ganda, D.R., \& Boruchovitch, E.; (2015). Self-handicapping strategies for learning of preservice teachers. Revista Estudos de Psicologia, 32(2), 417425.

Ganda, D. R. \& Boruchovitch, E. (2018). Promoting self-regulated learning of brazilian preservice student teachers: results of an intervention program. Frontiers in Education, 3(5), 1-12.

Ganda, D. R. \& Boruchovitch, E. (2019). Como promover a autorregulação da aprendizagem de futuros professores: Descrição de um programa no ensino superior. In E. Boruchovitch e M. A. M. Gomes. (Eds). Aprendizagem autorregulada: Como promovê-la no contexto educativo. Petrópolis, RJ: Vozes.

Glava, C., \& Glava, A. (2010). Teaching skills training trough e-learning. Procedia Social and Behavioral Sciences, 2, 1752-1756.

Goh, T., Seet B., \& Chen, N. (2012). The impact of persuasive SMS on students' self-regulated learning. British Journal of Educational Technology, 43(4), 624-640.

Gomes, M. A. M., \& Boruchovitch, E. (2011). Aprendizagem autorregulada da leitura: Resultados positivos de uma intervenção psicopedagógica. Psicologia, 27, 291-299. doi:10.1590/S0102-37722011000300004 
Granville, S., \& Dison, L. (2005). Thinking about thinking: Integrating selfreflection into an academic literacy course. Journal of English for Academic Purposes, 4, 99-118.

Hofer, B., Yu., S., \& Pintrich, P. (1998). Teaching college students to be selfregulated learners. In D. Schunk, \& B. Zimmerman (Eds.), Self-regulated learning: From teaching to self-reflective practice (pp. 57-85). New York, NY: Guilford Press.

Johnson, T., Archibald, T., \& Tenenbaum, G. (2010). Individual and team annotation effects on students' reading comprehension, critical thinking, and meta-cognitive skills. Computers in Human Behavior, 26, 1496-1507.

Kauffman, D. F., Zhao R., \& Yang, Y. S. (2011). Effects of online note taking formats and self-monitoring prompts on learning from online text: Using technology to enhance self-regulated learning. Contemporary Educational Psychology, 36(4), 313-322.

Kostons, D., Gog, T., \& Paas, F. (2012). Training self-assessment and taskselection skills: A cognitive approach to improving self-regulated learning. Learning and Instruction, 22, 121-132.

Lee, H. W., Lim, K. Y., and Grabowski, B. L. (2010). Improving self-regulation, learning strategy use, and achievement with metacognitive feedback. Educational Technology Research and Development, 58, 629-648. doi: $10.1007 /$ s11423-0109153-6

Leutner, D., Leopold, C., \& Elzen-Rump, V. (2007). Self-regulated learning with a text-highlighting strategy: A training experiment. Journal of Psychology, 215(3), 174-182.

Lizarraga, M.L., Baquedano, M.T., Mangado, T.C., \& Cardelle-Elawar, M. (2012). Enhancement of thinking skills: Effects of two intervention methods. Thinking Skills and Creativity, 4, 30-43.

Magalhães, C.R. (2012). A autorregulação da aprendizagem em programa institucional de acolhimento e suporte ao aluno universitário: Os professores como parceiros. Cadernos de Educação, 42, 143 - 167.

Martini, M.L., \& Del Prette, Z.A.P. (2002.) Atribuições de Causalidade para o sucesso e fracasso escolar dos seus alunos por professores do ensino fundamental. Interação em Psicologia, 6(2), 149-156.

Martini, M., \& Del Prette, Z. (2009). Crenças docentes e implicações para o processo de ensino-aprendizagem. Revista Semestral da Associação Brasileira de Psicologia Escolar e Educacional, 13, 75-85.

Marini, J., and Boruchovitch, E. (2014). Estratégias de Aprendizagem de Alunos Brasileiros do Ensino Superior: Considerações sobre adaptação, sucesso acadêmico e aprendizagem autorregulada. Revista Eletrônica de Psicologia Educação e Saúde 1, 102-126. 
Matsumoto, H., Hiromori, T., \& Nakayama, A. (2013). Toward a tripartite model of $\mathrm{L} 2$ reading strategy use, motivations, and learner beliefs. System, 41, 38-49.

McKeachie, W. J., Lin, Y. G., \& Middleton, M. J. (2004). Two types of low testanxious (low-worry) students. Coun. Clinical Psychoogy Journal, 1, 141152.

McKeachie, W. J., \& Svinicki, M. (2006). Teaching tips: Strategies, research, and theory for college and university teachers, 12ed. Boston, MA: Houghton Mifflin College.

Middleton, M., Abrams, E., \& Seaman, J. (2011). Resistance and disidentification in reflective practice with preservice teaching interns. New Direction Teaching and Learning, 126, 67-75. doi:10.1002/tl.445

Nuckels, M., Hubner, S., \& Renkl, A. (2009). Enhancing self-regulated learning by writing learning protocols. Learning and Instruction, 19, 259-271

Perels, F., Gurtler, T., \& Schmitz, B. (2005). Training of self-regulatory and problem-solving competence. Learning and Instruction, 15, 123-139.

Peters, E., \& Kitsantas, A. (2009). Self-regulation of student epistemic thinking in science: The role of metacognitive prompts. Educational Psychology, 30(1), 27-52.

Pozo, J. (1996). Estratégias de aprendizagem. In C. Coll, J. Palácios, \& A. Marchesi (eds.), Desenvolvimento psicológico e educação: Psicologia da educação (p. 176-197). Porto Alegre, RS: Artes Médicas.

Punhagui, G. C., \& Souza, N. A. (2012). Autorregulação na aprendizagem de língua inglesa: Ações e processos por meio de instrumentos autoavaliativos. Anais da I Fórum de Professores de Didática do Estado do Paraná.

Randi,J., Corno,L., \& Johnson,E. (2011). Transitioning from college classroom to teaching career: Self-regulation in prospective teachers. New Directions for Teaching and Learning, 126, 89-98.

Redford, J.; Thiede, K; Wiley, J.; Griffin, T. (2012). Concept mapping improves metacomprehension accuracy among 7th graders. Learning and Instruction, 22, 262-270.

Roque, S. C. F. (2012). Autorregulação em crianças do Pré-Escolar e do $1^{\circ}$ Ciclo do Ensino Básico Relatório de Estágio. (Dissertação de Mestrado). Universidade dos Açores, Departamento de Ciências da Educação, Portugal.

Rosário, P., Barbosa, J., Nuñez, J., \& Gonzalez-Pienda, J. (2005). Auto-regulação da aprendizagem a partir de estórias: Investigação no pré-escolar. Actas do $1^{0}$ Congresso Internacional de Aprendizagem na educação de Infância. CIANEI, Porto, Portugal.

Rosário, P., González-Pienda, J., Núnez, J. C., \& Mourão, R. (2005). Mejora del proceso de estudio y aprendizaje mediante la promoción de los procesos de autorregulación en estudiantes de enseñanza primaria y secundaria. Revista de Psicología y Educación, 1(2), 51-65. 
Rosário, P., Núnez, J., \& González-Pienda, J. (2006). Comprometer-se com o estudar na Universidade: "Cartas do Gervásio ao seu Umbigo". Almedina: Portugal.

Rosário, P., Mourão, R., Núñez, J. C., González-Pienda, J. A., Solano, P., \& Valle, A. (2007). Evaluating the efficacy of a program to enhance college students' self-regulation learning processes and learning strategies. Psicothema, 19, 422-427.

Rosário, P., Mourão, R., Núñez, J. C., González-Pienda, J. A., \& Solano, P. (2008). Storytelling as a promoter of self-regulated learning (SRL) throughout schooling. In A. Valle, J. C. Núñez, R. G., Cabanach, J. A. González-Pienda, \& S. Rodríguez (Eds.), Handbook of instructional resources and their applications in the classroom (pp. 107-122). New York, NY: Nova Science.

Rosário, P., Núñez, J. C., González-Pienda. J., Valle A., Trigo, L., \& Guimarães, C. (2010). Enhancing self-regulation and approaches to learning in first-year college students: a narrative based programme assessed in the Iberian Peninsula. European Journal Psychology Education, 25, 411-428.

Salonen, P., Vauras, M., \& Efklides, A. (2005). Social interaction: What can it tell us about metacognition and co-regulation in learning? European Psychologist, 10, 199-205.

Schunk, D. H., \& Zimmerman, B. J. (2008). Motivation and self-regulated learning: Theory, research, and applications. New Jersey: Lawrence Erlbaum Associates.

Shen, H. \& Liu, W. (2011). A survey on the self-regulation efficacy in DUT's english blended learning context. Journal of Language Teaching and Research, 2(5), 1099-1110.

Simpson, M. L., Hynd, C. R, Nist, S. L., \& Burrell, K. (1997). College academic assistance programs and practices. Educational Psychology Review, 9,3987.

Sitzmann, T., Bell, B.S., Kraiger, K., \& Kanar, A. (2009). A multilevel analysis of the effect of prompting self-regulation in technology delivered instruction. Personnel Psychology, 62, 697- 734.

Sitzmann, T. \& Ely, K. (2010). Sometimes you need a reminder: The effects of prompting selfregulation on regulatory processes, learning, and attrition. Journal of Applied Psychology, 95(1), 132-144.

Sitzmann, T. \& Johnson, S. K. (2012). The best laid plans: Examining the conditions under which a planning intervention improves learning and reduces attrition. Journal of Applied Psychology, 97(5), 967-981.

Sun, J. C.-Y., \& Rueda, R. (2012). Situational interest, computer self-efficacy and self-regulation: Their impact on student engagement in distance education. British Journal of Educational Technology, 43(2), 191-204. doi: 10.1111/j.1467-8535.2010.01157.x 
VanderStoep, S., \& Pintrich, P. (2003). Learning to Learn: The Skill and Will of College Success. Upper Saddle River, NJ: Prentice Hall.

Warfield, J., Wood, T., \& Lehman, J. D. (2005). Autonomy, beliefs and the learning of elementary mathematics teachers. Teaching and Teacher Education, 21(4), 439-459. doi:10.1016/j.tate.2005.01.011

Weiner, B. (2010). The development of an attribution-based theory of motivation: a history of ideas. Educational Psychology 45, 28-36. doi: $10.1080 / 00461520903433596$

Wolters, C. (2010). Self-Regulated Learning and the 21st Century Competences. Houston: Department of Educational Psychology, University of Houston, 127.

Woolfson, L.M., \& Brady, K. (2009). An investigation of factors impacting on mainstream teachers'beliefs about teaching students with learning difficulties. Educational Psychology 29(2), 221-238.

Zimmerman, B. J., Bonner, S., \& Kovach, R. (2002). Understanding the principles of self-regulated learning. In Developing self-regulated learners: beyond achievement to self-efficacy. Washington, American Psychological Association, pp.5-24.

Zimmerman, B.J., \& Schunk, D. H. (2011). Handbook of self-regulation of learning and performance. New York, NY: Taylor \& Francis.

Zimmerman, B.J. \& Tsikalas, K.E. (2005) Can computer-based learning environments (CBLEs) be used as self-regulatory tools to enhance learning? Educational Psychologist, 40, 267-271. doi: 10.1207/s15326985ep4004_8

Zimmerman, B. J., \& Schunk, D. H. (2008). Motivation: An essential dimension of self-regulated learning. In D. H. Schunk, \& B. J. Zimmerman, Motivation and self-regulated learning: Theory, research, and applications. England: MPG Books Group, 1-30.

\section{DECLARAÇÃO DE CONFLITOS DE INTERESSE}

As autoras declaram que a pesquisa foi conduzida na ausência de quaisquer relações comerciais ou financeiras que poderiam se constituir como potencial conflito de interesse. 
Sobre as autoras

Danielle Ribeiro Ganda é psicóloga pela Universidade Federal de Uberlândia/UFU, mestre e doutora em Educação pela Universidade Estadual de

Campinas/UNICAMP. Pesquisadora colaboradora do Grupo de Estudos e Pesquisas em Psicopedagogia - GEPESP da Universidade Estadual de Campinas/UNICAMP. danielleganda@hotmail.com

Evely Boruchovitch é psicóloga pela Universidade Estadual do Rio de Janeiro UERJ, doutora pela University of Southern California. Trabalha na linha Psicologia Escolar e Educacional na Universidade Estadual de Campinas/UNICAMP. É bolsista de produtividade 1-B do CNPq. evely@unicamp.br

As autoras agradecem a Fundação de Amparo à Pesquisa do Estado de São Paulo - FAPESP pelo financiamento da pesquisa e a segunda autora agradece também ao CNPq.

Recebido em: 11/12/2019

Aceito em: $16 / 12 / 2019$ 